\title{
Allee effects in a predator-prey system with a saturated recovery function and harvesting
}

\author{
Mohammad Javidi* and Nemat Nyamorady \\ Department of Mathematics, Faculty of Sciences Razi University, 67149 Kermanshah, Iran \\ ${ }^{*}$ Corresponding author E-mail: mo_javidi@yahoo.com
}

\begin{abstract}
In this paper, we will consider the Allee effects on predator-prey system with a saturated recovery function and harvesting. Local stability analysis of biologically feasible equilibrium points is worked out with help of ecological as well as disease basic reproduction numbers. We proved that the equilibrium $P_{0}=(0,0)$ of the predator-prey system is (i) a saddle point in weak Allee effects (WAE) and (ii) asymptotically stable in strong Allee effects (SAE). We proved that the equilibrium $P_{1}=(\beta, 0)$ of the system is a saddle point if $R_{0}(1)<1$ and unstable if $R_{0}(1)>1$ in SAE case. Also we proved that the equilibrium $P_{2}=(1,0)$ of the system is a saddle point if $R_{0}(1)>1$ and asymptotically stability if $R_{0}(1)<1$ in SAE case. It is shown that the coexistence equilibria is not asymptotically stable. Numerical simulations are carried out for a hypothetical set of parameter values to substantiate our analytical findings.
\end{abstract}

Keywords: Dynamic analysis, local stability, simulation, reproduction number.

\section{Introduction}

In this paper, we will consider predator-prey system with a saturated recovery function and harvesting. In recent decades, population models appearing in various fields of mathematical biology have been proposed and studied extensively due to their universal existence and importance [1]. Among the most widely used population models in theoretical ecology, the Holling-Tanner model plays a special role in view of the interesting dynamics it possesses. This model has been widely studied by several researchers, for instance $[2,3,4,5,6,7]$.

The most crucial element in prey-predator models is the "functional response" or "trophic function", the function that describes the number of prey consumed per predator per unit time for given quantities of prey and predator. Various forms of functional responses have become the focus of considerable attention from time to time in ecological literature. The most important and useful functional response is the so-called Michaelis-Menten or Holling type-II functional response of the form $V(x)=\frac{c x}{m+x}$, where $\mathrm{x}$ and $\mathrm{y}$ are the population densities of the prey and predator, respectively; $c$ is the maximal predator per capita consumption rate, i.e. the maximum number of prey that can be eaten by a predator in each time unit and $\mathrm{m}$ is the half capturing saturation constant, i.e. the number of prey necessary to achieve one-half of the maximum rate c. Many species models with such functional responses are extensively studied in ecological literature $[8,9,10]$.

In [11], a modified Holling-Tanner predator-prey model with time delay is considered. By regarding the delay as the bifurcation parameter, the local asymptotic stability of the positive equilibrium is investigated. In [12], a delayed stage-structured predator-prey model with non-monotone functional responses is proposed. It is assumed that immature individuals and mature individuals of the predator are divided by a fixed age, and that immature predators do not have the ability to attack prey. In [13], the main feature is that the authors introduce time delay and pulse into the predator-prey (natural enemy-pest) model with age structure, exhibit a new modeling method which is applied to investigate impulsive delay differential equations, and give some reasonable suggestions for pest management. The authors of [14], have studied changes in the dynamics of a predator population, which otherwise lives on a native prey, in presence of migratory prey that carries some infection. They study predicts one of the four behaviors when some system parameters were varied: eradication of the disease, predator extinction, coexistence 
at stable equilibrium, or coexistence on limit cycles.

Lotka [15] in 1925 and Volterra [16] in 1926 introduced the first predator-prey model. After that many more complicated but realistic PP models have been formulated by ecologists and mathemati-cians. One of the most popular prey-predator models was introduced by Freedman in 1980 (see [17] for more details), which has the MichaelisMenten type functional response. The authors of [18], studied a simple prey-predator interaction where predator population is subject to harvesting.

It has been shown in a large number of theoretical works that replacing the logistic growth function with an Allee-type function having a threshold (the strong Allee effect) could dramatically change the patterns of dynamics $([19,20,21,22,23,24]$. Interestingly, even in the case where the Allee effect is weak, the model predictions on ecosystem behaviour can be rather different compared to the same systems with logistic growth [25, 26, 27]. As such, the presence of the Allee effect (weak or strong) should also seriously alter previous theoretical findings on the ratio-dependent predatorprey model with logistic growth. Thus there is an urgent need for mathematical analysis of the behaviour of ratio-dependent predator-prey models with more realistic growth rate functions including the Allee effect for prey species.

The authors of [28], studied a ratio-dependent predator-prey model with the Allee effect in the growth of the prey population. They analyse the stability properties of the system, present a complete bifurcation analysis and show all possible non-degenerated phase portraits. In this paper, we consider a predator-prey system with a saturated recovery function and harvesting with Allee effects as the following form

$$
\left\{\begin{array}{l}
\frac{d W(t)}{d t}=W(t)(W(t)-\beta)(1-W(t))-\alpha \frac{W(t) V(t)}{1+\alpha_{1} W(t)}, \\
\frac{d V(t)}{d t}=\gamma \frac{W(t) V(t)}{1+\alpha_{1} W(t)}-d_{0} V(t)-E V(t),
\end{array}\right.
$$

with the following initial conditions: $W(0)=W_{0}>0, V(0)=V_{0}>0$.

Let $W(t)$ and $V(t)$ be the prey and predator densities at time $t$ respectively. Let $d_{0}$ be the food-independent death rate and $\alpha, \alpha_{1}, d_{0}, \beta, E$ are positive real numbers. Modelling a strong Allee effect (SAE) implies $0<\beta \leq 1$, whereas a weak Allee effect (WAE) requires $-1<\beta \leq 0$.

The organization of this paper is as follows. In the next section, we present local stability of equilibrium. In Section 3 we present the numerical method. Also a brief discussion is given in Section 3.

\section{Main Results}

\subsection{Stability of equilibria}

In this section we deal with the local stability of the system (1). Let

$$
\frac{d W(t)}{d t}=0, \frac{d V(t)}{d t}=0 .
$$

The other equilibria of (2) are the points of intersections of the following two curves

$$
\left\{\begin{array}{l}
W(t)(W(t)-\beta)(1-W(t))=\alpha \frac{W(t) V(t)}{1+\alpha_{1} W(t)}, \\
\beta \frac{W(t) V(t)}{1+\alpha_{1} W(t)}=\left(d_{0}+E\right) V(t) .
\end{array}\right.
$$

Solving (3) for its roots, we can get that system (1) has four equilibria points as follows:

1. The prey-predator free equilibria $P_{0}=(0,0)$.

2. The predator free equilibria $P_{1}=(\beta, 0)$.

3. The predator free equilibria $P_{2}=(1,0)$.

4. The coexistence equilibria $P_{3}=\left(W^{*}, V^{*}\right)$ where

$$
\begin{aligned}
& W^{*}=\frac{d_{0}+E}{\gamma-\alpha_{1}\left(d_{0}+E\right)}=\frac{1}{R_{0}(1)+\alpha_{1}\left(R_{0}(1)-1\right)}, \\
& V^{*}=\frac{1}{\alpha} W^{*}\left(W^{*}-\beta\right)\left(1-W^{*}\right)\left(1+\alpha_{1} W^{*}\right)
\end{aligned}
$$




$$
=\frac{R_{0}(1)\left(R_{0}(1)-1\right)\left(1+\alpha_{1}\right)^{2}}{\alpha\left(R_{0}(1)+\alpha_{1}\left(R_{0}(1)-1\right)\right)^{3}}\left(R_{0}(1)\left(1+\alpha_{1}\right)-\left(\alpha_{1}-\beta\right)\right)
$$

where $R_{0}(\beta)=\frac{\gamma \beta}{\left(1+\alpha_{1} \beta\right)\left(d_{0}+E\right)}$ is the basic reproduction number. The existence criteria for the interior equilibrium point $P_{3}$ is $\mathbf{H 1}: R_{0}(1)>\frac{\alpha_{1}}{\alpha_{1}+1}, \mathbf{H 2}: R_{0}(1) \leq \frac{1+\alpha_{1} \beta}{\left(\alpha_{1}+1\right) \beta}$. The next crucial question is about the stability of the above equilibria. The local stability analysis of the other equilibria is more straightforward and can be done based upon the standard linearization technique and using the Jacobian matrix. The Jacobian matrix of system (1) at the equilibrium point $(W, V)$ is

$$
J(W, V)=\left(\begin{array}{cc}
(W-\beta)(1-W)+W(1+\beta-2 W)-\frac{\alpha V}{\left(1+\alpha_{1} W\right)^{2}} & -\frac{\alpha W}{1+\alpha_{1} W(t)} \\
\frac{\gamma V}{\left(1+\alpha_{1} W\right)^{2}} & \frac{\gamma W \alpha_{0} W}{1+\alpha_{1}}-E
\end{array}\right) .
$$

The Jacobian evaluated at $P_{0}$ is given by

$$
J\left(P_{0}\right)=\left(\begin{array}{cc}
-\beta & 0 \\
0 & -d_{0}-E
\end{array}\right),
$$

with the characteristic equation

$$
Q(\lambda)=\operatorname{det}\left(\lambda-J\left(P_{0}\right)\right)=(\lambda+\beta)\left(\lambda+d_{0}+E\right) .
$$

The eigenvalues corresponding to the equilibrium $D_{0}$ are

$$
\lambda_{1}=-\beta, \lambda_{2}=-d_{0}-E .
$$

Then we have $\lambda_{1}<0$ in SAE and $\lambda_{1}>0$ in WAE and $\lambda_{2}<0$. Consequently, we have the following theorem:

Theorem 1. The equilibrium $P_{0}$ of system (1) is

(i) a saddle point in WAE.

(ii) asymptotically stable in SAE.

Now we investigate the asymptotically stability of system (1) at the equilibrium point $P_{1}$ only in SAE case. In a similar manner, the Jacobian evaluated at $P_{1}$ is given by

$$
J\left(P_{1}\right)=\left(\begin{array}{cc}
\beta(1-\beta) & -\frac{\alpha \beta}{1+\alpha_{1} \beta} \\
0 & \frac{\gamma \beta}{1+\alpha_{1} \beta}-d_{0}-E
\end{array}\right),
$$

with the characteristic equation

$$
Q(\lambda)=\operatorname{det}\left(\lambda-J\left(P_{1}\right)\right)=(\lambda-\beta(1-\beta))\left(\lambda-\frac{\gamma \beta}{1+\alpha_{1} \beta}+d_{0}+E\right) .
$$

The eigenvalues corresponding to the equilibrium $P_{1}$ are

$$
\lambda_{1}=\beta(1-\beta), \lambda_{2}=\frac{\gamma \beta}{1+\alpha_{1} \beta}-d_{0}-E .
$$

Then we have $\lambda_{1}>0$ and $\lambda_{2}<0$ if $R_{0}(\beta)<1$ and $\lambda_{2}>0$ if $R_{0}(\beta)>1$. Consequently, we have the following theorem:

Theorem 2. The equilibrium $P_{1}$ of system (1) is a saddle point if $R_{0}(1)<1$ and unstable if $R_{0}(1)>1$ in $S A E$ case.

Now we investigate the asymptotically stability of system (1) at the equilibrium point $P_{2}$. In a similar manner, the Jacobian evaluated at $P_{2}$ is given by

$$
J\left(P_{2}\right)=\left(\begin{array}{cc}
\beta-1 & -\frac{\alpha \beta}{1+\alpha_{1}} \\
0 & \frac{\gamma}{1+\alpha_{1}}-d_{0}-E
\end{array}\right),
$$

with the characteristic equation

$$
Q(\lambda)=\operatorname{det}\left(\lambda-J\left(P_{2}\right)\right)=(\lambda+1-\beta)\left(\lambda-\frac{\gamma \beta}{1+\alpha_{1}}+d_{0}+E\right) .
$$


The eigenvalues corresponding to the equilibrium $P_{2}$ are

$$
\lambda_{1}=\beta-1, \lambda_{2}=\frac{\gamma}{1+\alpha_{1}}-d_{0}-E .
$$

Then we have $\lambda_{1}<0$ and $\lambda_{2}<0$ if $R_{0}(1)<1$ and $\lambda_{2}>0$ if $R_{0}(1)>1$. Consequently, we have the following theorem:

Theorem 3. The equilibrium $P_{2}$ of system (1) is a saddle point if $R_{0}(1)>1$ and asymptotically stability if $R_{0}(1)<1$ in $S A E$ case.

Next we consider the stability of the interior equilibria under the restrictions H1. Now we consider the asymptotically stability of system (2) at the equilibrium point $P_{3}$. The Jacobian matrix is of the form

$$
\begin{aligned}
J\left(P_{3}\right) & =\left(\begin{array}{cc}
\left(W^{*}-\beta\right)\left(1-W^{*}\right)+W^{*}\left(1+\beta-2 W^{*}\right)-\frac{\alpha V^{*}}{\left(1+\alpha_{1} W^{*}\right)^{2}} & -\frac{\alpha W^{*}}{1+\alpha_{1} W^{*}} \\
\frac{\gamma V^{*}}{\left(1+\alpha_{1} W^{*}\right)^{2}} & \frac{\gamma W^{*}}{1+\alpha_{1} W^{*}}-d_{0}-E
\end{array}\right) \\
& =\left(\begin{array}{cc}
\frac{\alpha_{1}}{\gamma}\left(W^{*}-\beta\right)\left(1-W^{*}\right)\left(d_{0}+E\right)+W^{*}\left(1+\beta-2 W^{*}\right) & -\frac{\alpha\left(d_{0}+E\right)}{\gamma} \\
\frac{1}{\alpha W^{*}}\left(W^{*}-\beta\right)\left(1-W^{*}\right)\left(d_{0}+E\right) & 0
\end{array}\right)
\end{aligned}
$$

Then we have

$$
\begin{gathered}
\operatorname{det}\left(J\left(P_{3}\right)\right)=\frac{1}{\gamma W^{*}}\left(W^{*}-\beta\right)\left(1-W^{*}\right)\left(d_{0}+E\right)^{2}, \\
\operatorname{Tr}\left(J\left(P_{3}\right)\right)=\frac{\alpha_{1}}{\gamma}\left(W^{*}-\beta\right)\left(1-W^{*}\right)\left(d_{0}+E\right)+W^{*}\left(1+\beta-2 W^{*}\right) .
\end{gathered}
$$

It is clear that $\operatorname{det}\left(J\left(P_{3}\right)\right)>0$ and $\operatorname{Tr}\left(J\left(P_{3}\right)\right)>0$. Therefore the coexistence equilibria $P_{3}$ is not asymptotically stable.

\section{Numerical Simulation and discussion}

In this section, to verify the effectiveness of the obtained results, some numerical simulations for the fractional-order prey-predator system (1) have been conducted. All the differential equations are solved using the method proposed in the previous section. In all numerical runs, the solution has been approximated at $\Delta t=0.01$. In Figs. 1 , we display phase portrait of system (1). In this case we consider SAE with $\beta=0.001$. The values of parameters are $\alpha=0.1, \gamma=0.4, E=0.1, \alpha_{1}=0.1$ with the initial conditions

$\left[W_{0}, V_{0}\right]=[0.7,1.2],[0.8,0.5],[0.3,3.4],[0.9 .2 .3]$ for various values of $R_{0}$.

In Figs. 2, we display phase portrait of system (1). In this case we consider SAE with $\beta=0.1$. The values of parameters are $\alpha=0.1, \gamma=0.9, d_{0}=0.15, \alpha_{1}=0.1$ with the initial conditions

$\left[W_{0}, V_{0}\right]=[0.5,2.2],[0.9,2.5],[0.3,1.4],[0.7,3.2]$ for various values of $E$ and $R_{0}<1$.

In Figs. 3, we display phase portrait of system (1). In this case we consider SAE with $\beta=1$. The values of parameters are $\alpha=0.1, \gamma=0.9, d_{0}=0.1, \alpha_{1}=0.1$ with the initial conditions

$\left[W_{0}, V_{0}\right]=[0.5,2.2],[0.9,1],[0.3,1.4],[0.7,3.2]$ for various values of $E$ and $R_{0}>1$.

In Figs. 4, we display phase portrait of system (1). In this case we consider WAE with $\beta=-0.1$. The values of parameters are $\alpha=0.1, d_{0}=0.14, E=0.2, \alpha_{1}=0.1$ with the initial conditions

$\left[W_{0}, V_{0}\right]=[0.3,3.5],[0.9,1],[0.3,1.4],[0.9 .3 .4]$ for various values of $\gamma$.

In Figs. 5, we display phase portrait of system (1). In this case we consider WAE with $\beta=-0.9$. The values of parameters are $\alpha=0.1, d_{0}=0.14, E=0.2, \alpha_{1}=0.1$ with the initial conditions

$\left[W_{0}, V_{0}\right]=[0.3,3.5],[0.9,1],[0.3,1.4],[0.9 .3 .4]$ for various values of $\gamma$.

In Figs. 6, we display phase portrait of system (1). In this case we consider WAE with $\beta=-0.5$. The values of parameters are $\alpha=0.1, \gamma=5, d_{0}=0.5, \alpha_{1}=0.5$ with the initial conditions $\left[W_{0}, V_{0}\right]=[0.3,3.5],[0.7,3],[0.5,1.4],[0.9 .3 .4]$ for various values of $E$. 

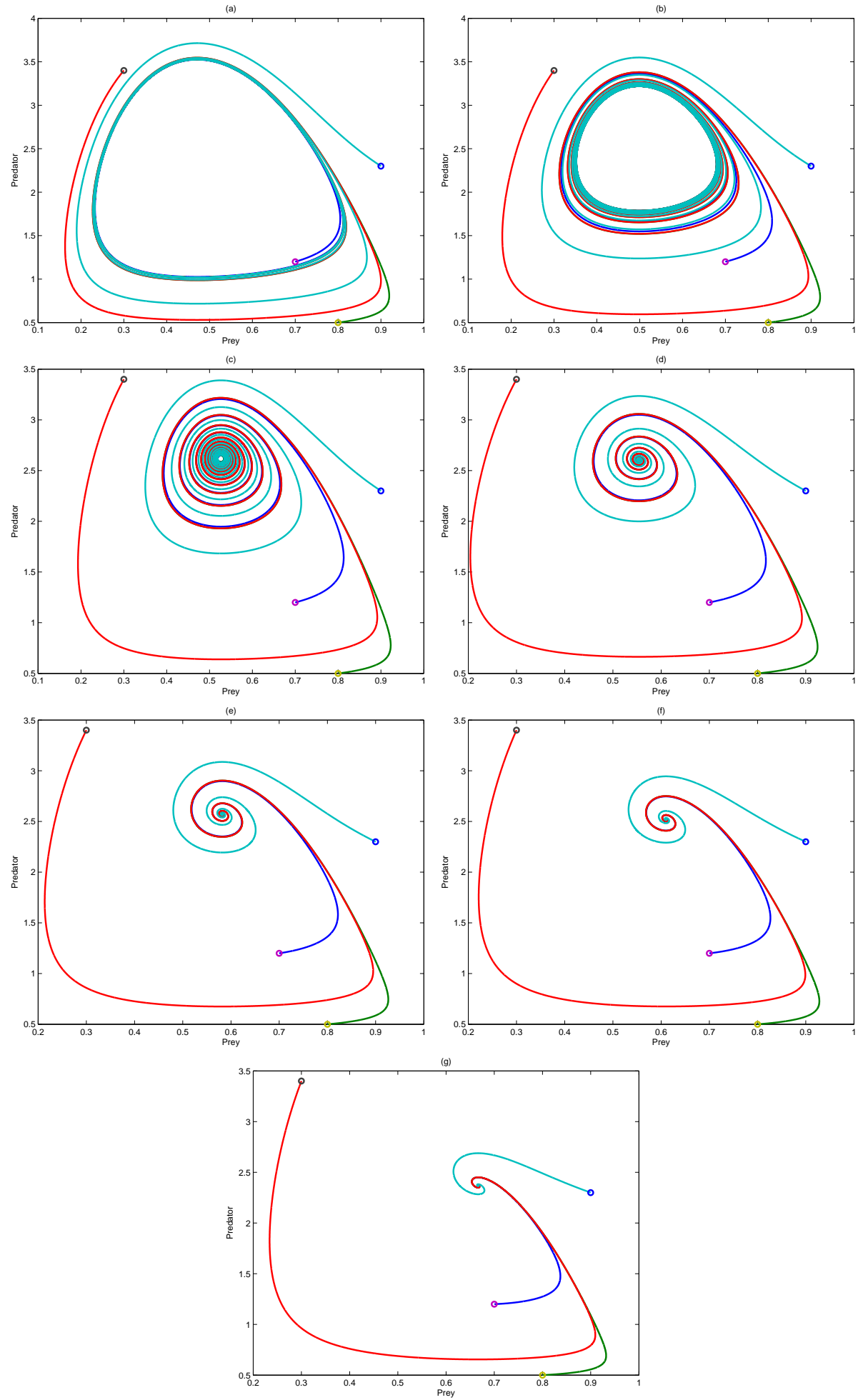

Figure 1: Phase portrait of system (1). In this case we consider SAE with $\beta=0.001$. The values of parameters are $\alpha=0.1, \gamma=0.4, E=0.1, \alpha_{1}=0.1$ with the initial conditions $\left[W_{0}, V_{0}\right]=[0.7,1.2],[0.8,0.5],[0.3,3.4],[0.9 .2 .3]$ for (a) $R_{0}=0.0022(\mathrm{~b}) R_{0}=0.0021(\mathrm{c}) R_{0}=0.0020(\mathrm{~d}) R_{0}=0.0019(\mathrm{e}) R_{0}=0.0018(\mathrm{f}) R_{0}=0.0017(\mathrm{~g}) R_{0}=0.0016$ 

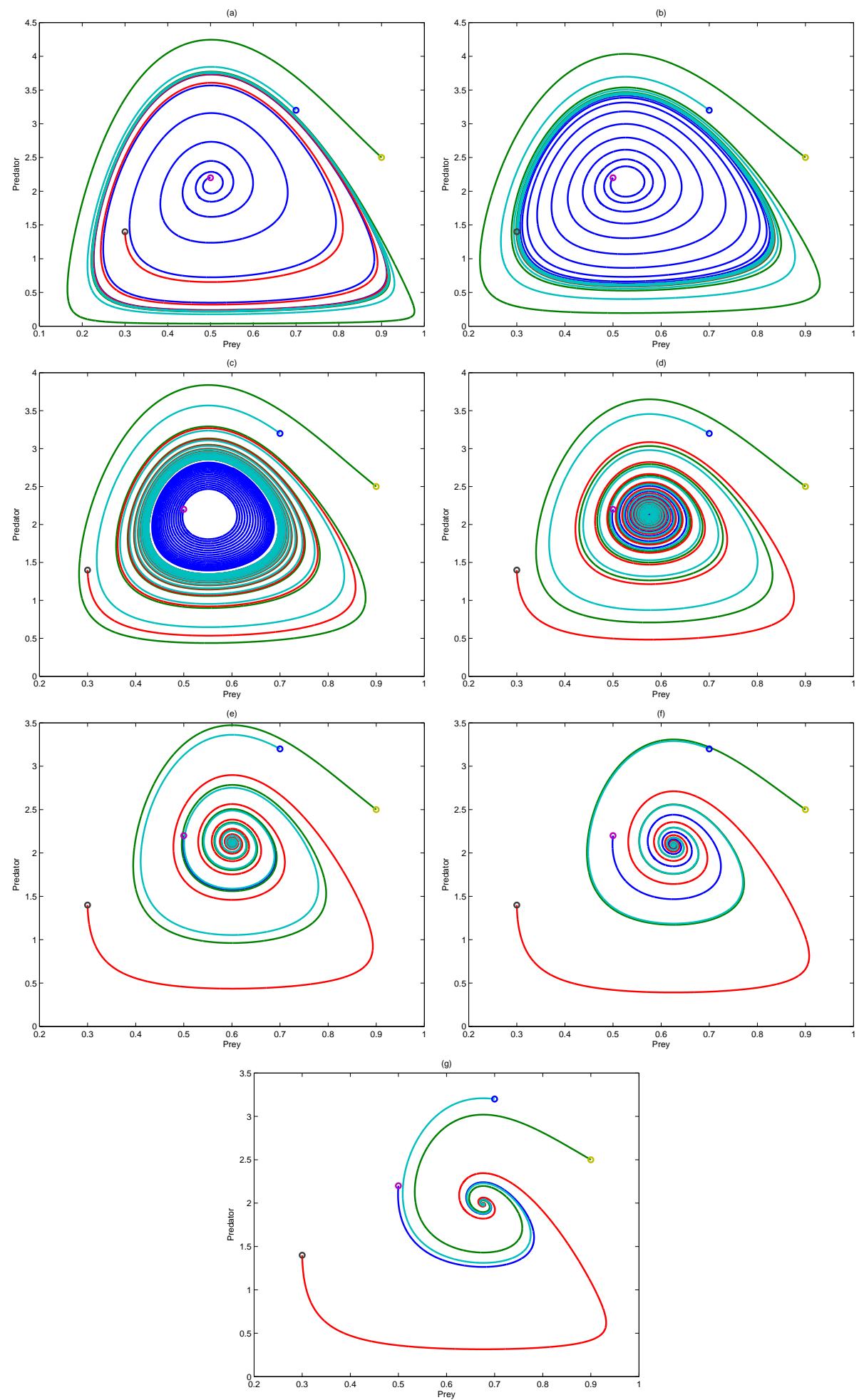

Figure 2: Phase portrait of system (1). In this case we consider SAE with $\beta=0.1$. The values of parameters are $\alpha=0.1, \gamma=0.9, d_{0}=0.15, \alpha_{1}=0.1$ with the initial conditions $\left[W_{0}, V_{0}\right]=[0.5,2.2],[0.9,2.5],[0.3,1.4],[0.7,3.2]$ for for (a) $E=0.28$ (b) $E=0.30$ (c) $E=0.32$ (d) $E=0.34$ (e) $E=0.36$ (f) $E=0.38$ (g) $E=0.42$. 

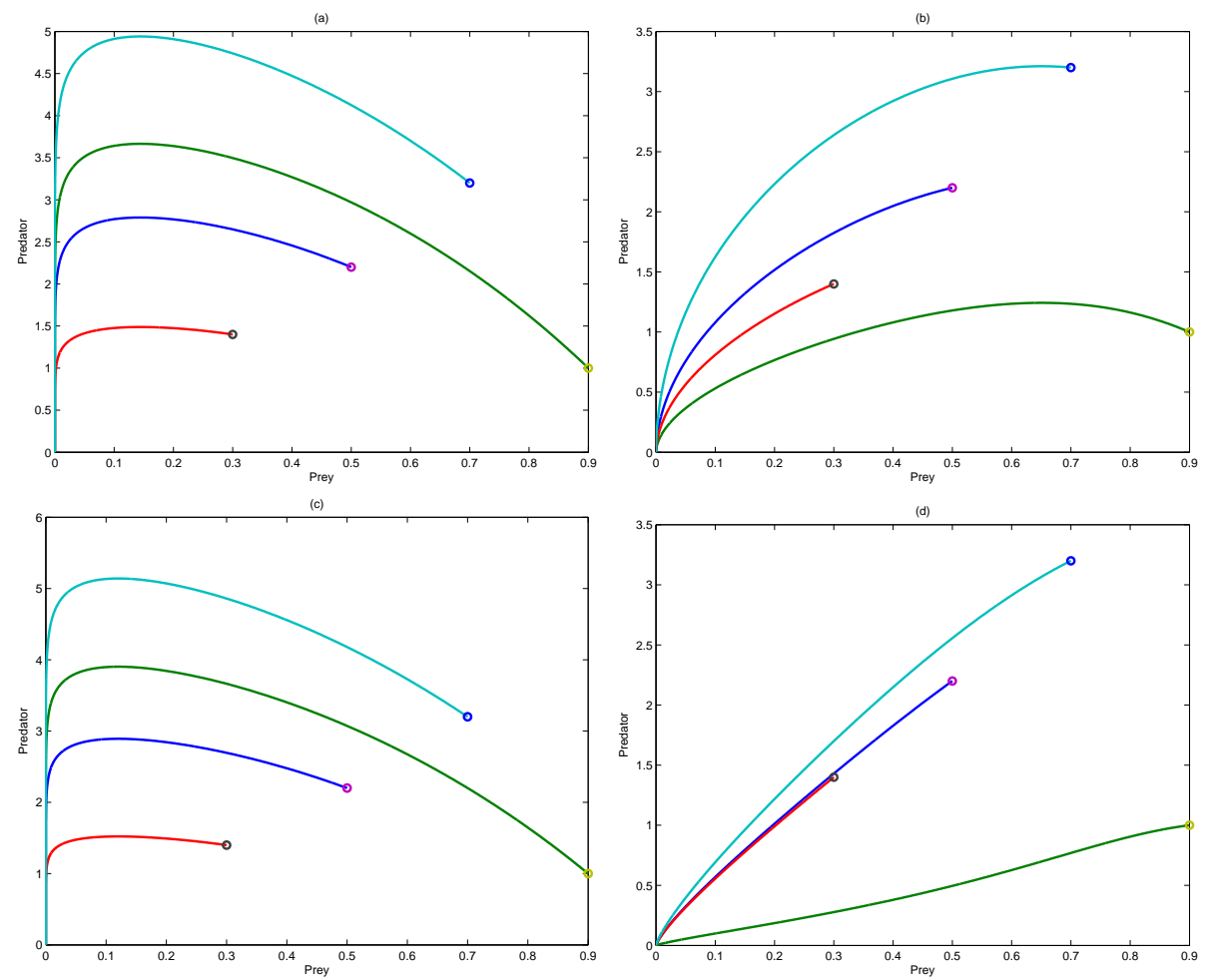

Figure 3: Phase portrait of system (1). In this case we consider SAE with $\beta=1$. The values of parameters are $\alpha=$ $0.1, \gamma=0.9, d_{0}=0.1, \alpha_{1}=0.1$ with the initial conditions $\left[W_{0}, V_{0}\right]=[0.5,2.2],[0.9,1],[0.3,1.4],[0.7,3.2]$ for for $(\mathrm{a}) E=0.028$ (b) $E=0.45$ (c) $E=0.007$ (d) $E=0.7$. 

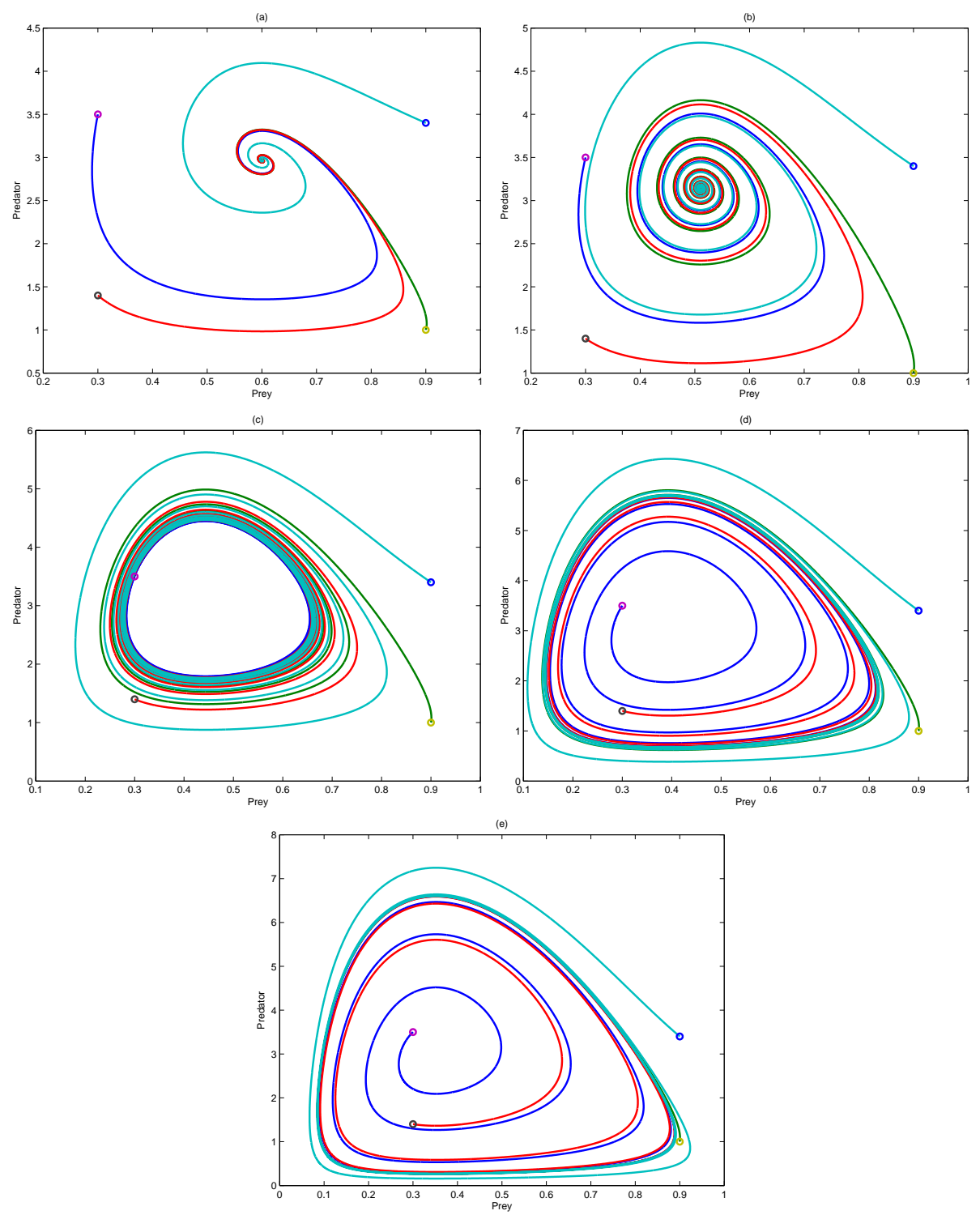

Figure 4: Phase portrait of system (1). In this case we consider WAE with $\beta=-0.1$. The values of parameters are $\alpha=0.1, d_{0}=0.14, E=0.2, \alpha_{1}=0.1$ with the initial conditions $\left[W_{0}, V_{0}\right]=[0.3,3.5],[0.9,1],[0.3,1.4],[0.9 .3 .4]$ for $(\mathrm{a}) \gamma=0.6$ (b) $\gamma=0.7$ (c) $\gamma=0.8$ (d) $\gamma=0.9$ (e) $\gamma=1$. 

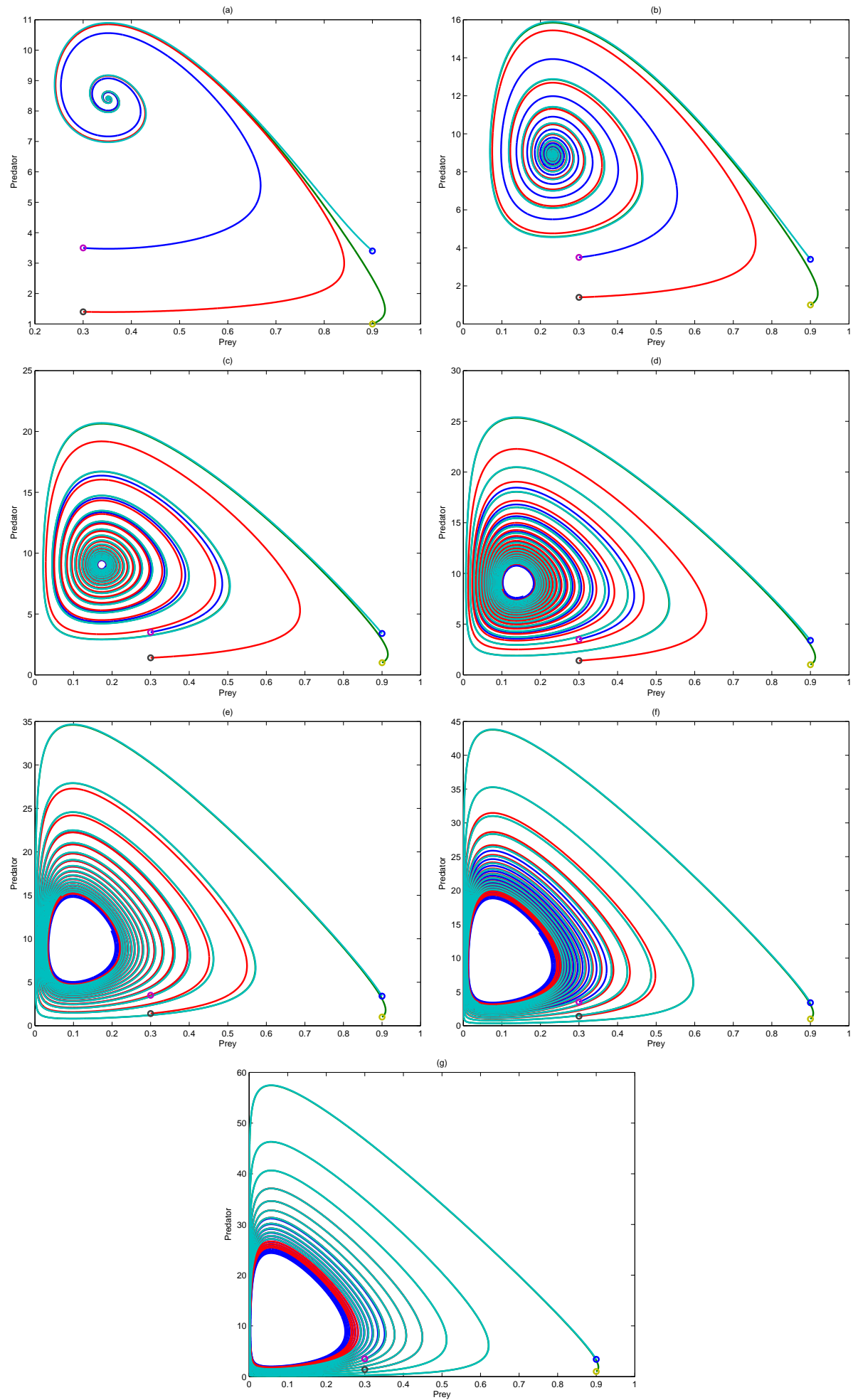

Figure 5: Phase portrait of system (1). In this case we consider WAE with $\beta=-0.9$. The values of parameters are $\alpha=0.1, d_{0}=0.14, E=0.2, \alpha_{1}=0.1$ with the initial conditions $\left[W_{0}, V_{0}\right]=[0.3,3.5],[0.9,1],[0.3,1.4],[0.9 .3 .4]$ for $(\mathrm{a}) \gamma=1$ (b) $\gamma=1.5(\mathrm{c}) \gamma=2(\mathrm{~d}) \gamma=2.5(\mathrm{e}) \gamma=3.5$ (f) $\gamma=4.5(\mathrm{~g}) \gamma=6$. 

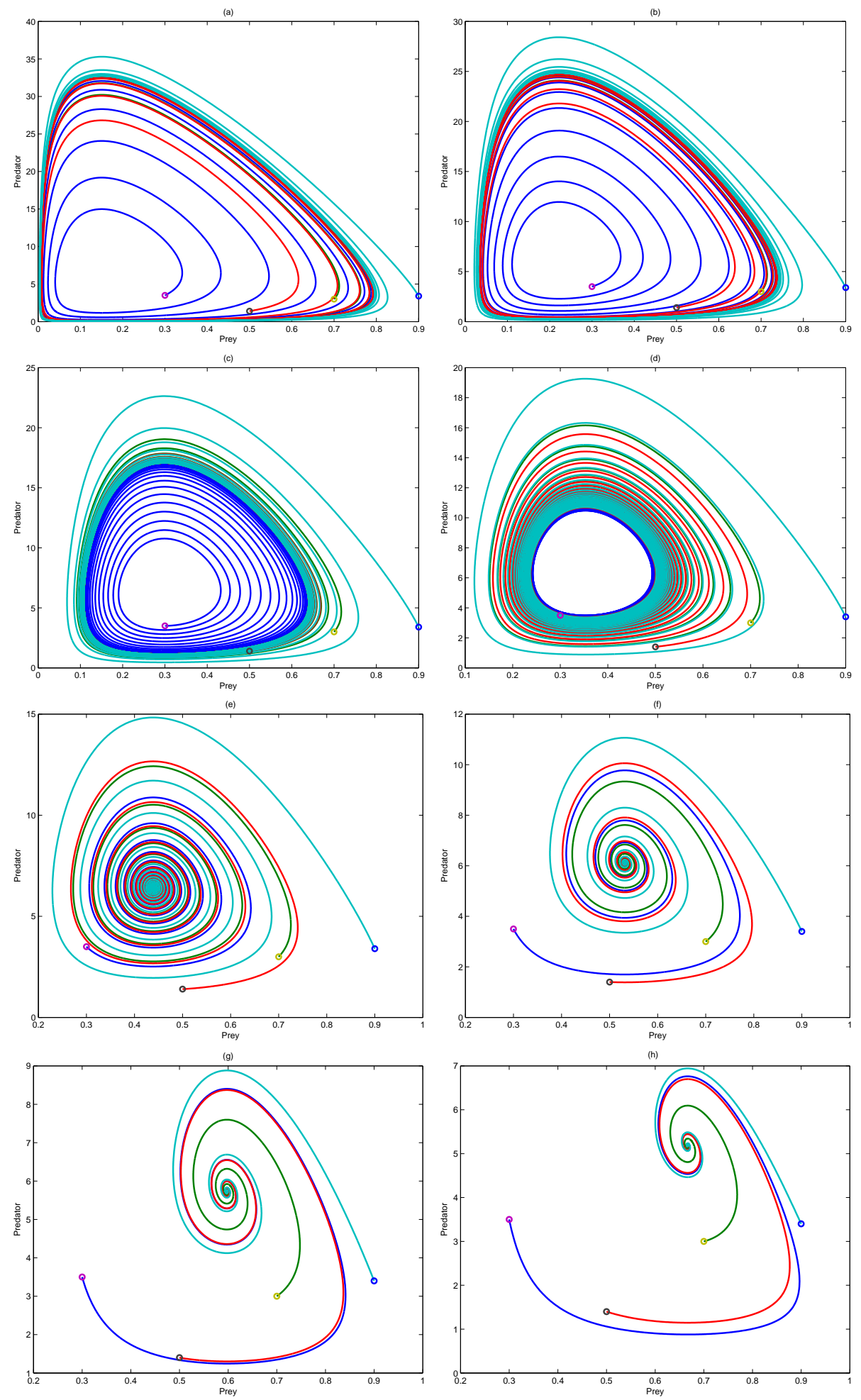

Figure 6: Phase portrait of system (1). In this case we consider WAE with $\beta=-0.5$. The values of parameters are $\alpha=0.1, \gamma=5, d_{0}=0.5, \alpha_{1}=0.5$ with the initial conditions $\left[W_{0}, V_{0}\right]=[0.3,3.5],[0.7,3],[0.5,1.4],[0.9 .3 .4]$ for (a) $E=0.2$ (b) $E=0.5$ (c) $E=0.8$ (d) $E=1$ (e) $E=1.3$ (f) $E=1.6(\mathrm{~g}) E=1.8$ (h) $E=2$. 


\section{References}

[1] A.A. Berryman, The origins and evolution of predator-prey theory, Ecology 73 (1992) 1530-1535.

[2] P.A. Braza, The bifurcations structure for the Holling Tanner model for predator-prey interactions using two-timing, SIAM J. Appl. Math. 63 (2003) 889-904.

[3] S.B. Hsu, T.W. Hwang, Global stability for a class of predator-prey systems, SIAM J. Appl. Math. 55 (1995) $763-783$.

[4] Z. Liang, H. Pan, Qualitative analysis of a ratio-dependent Holling-Tanner model, J. Math. Anal. Appl. 334 (2007) 954-964.

[5] M. Haquea, E. Venturino, The role of transmisible diseases in the Holling-Tanner predator-prey model, Theor. Popul. Biol. 70 (2006) 273-288.

[6] J.D. Murray, Mathematical Biology, Spring-Verlag, New York, 1993.

[7] J.T. Tanner, The stability and intrinsic growth rates of prey and predator populations, Ecology 56 (1975) 855-867.

[8] S. Ruan, D. Xiao, Global analysis in a predatorprey system with nonmonotonic functional response, SIAM J. Appl. Math. 61 (2001) 1445-1472.

[9] A. Maiti, G.P. Samanta, Deterministic and stochastic analysis of a prey-dependent predatorprey system, Int. J. Math. Educ. Sci. Technol. 36 (2005) 65-83.

[10] M. Bandyopadhyay, C.G. Chakrabarti, Deterministic and stochastic analysis of a non-linear preypredator system, J. Biol. Syst. 11 (2003) 161-172.

[11] J.-F. Zhang, Bifurcation analysis of a modified Holling-Tanner predator-prey model with time delay, Applied Mathematical Modelling 36 (2012) 1219-1231.

[12] Y. Xia, J. Cao, S. S. Cheng, Multiple periodic solutions of a delayed stage-structured predator-prey model with nonmonotone functional responses, Applied Mathematical Modelling 31 (2007) 1947-1959.

[13] X. Meng, J. Jiao, L. Chen, The dynamics of an age structured predator-prey model with disturbing pulse and time delays, Nonlinear Analysis: RealWorld Applications 9 (2008) 547-561.

[14] S. Chatterjee, et al., How population dynamics change in presence of migratory prey and predators preference. Ecol. Complex. (2012), doi:10.1016/j.ecocom.2012.03.001.

[15] A. Lotka, Elements of Physical Biology, Williams and Wilkins, Baltimore, 1925.

[16] V. Volterra, Variazioni e fluttuazioni del numero di individui in specie animali conviventi, Mem. Accd. Lincei. 2 (1926) 31-113.

[17] H. Freedman, Deterministic Mathematical Models in Population Ecology, Marcel Dekker, New York, 1980.

[18] S. Chakraborty, S. Pal, N. Bairagi, Predator-prey interaction with havesting: mathematical study with biological ramifications, Appl. Math. Modell. (2011), doi:10.1016/j.apm.2011.11.029.

[19] M.A. Lewis, P. Kareiva, Allee dynamics and the spread of invading organisms. Theor. Popul. Biol. 43 (1993), $141-158$.

[20] F. Courchamp, T. Clutton-Brock, B. Grenfell, Inverse density dependence and the Allee effect. TREE 14(1999) 405-410.

[21] A.Y. Morozov, S. Petrovoskii, B.L. Li, Spatiotemporal complexity of patchy invasion in a predator-prey system with the Allee effect. J. Theor. Biol. 238(2006) 18-35.

[22] L. Berec, E. Angulo, F. Courchamp, Multiple Allee effects and population management. TREE 22 (2007) $185-191$.

[23] F.M. Hilker, Population collapse to extinction: the catastrophic combination of parasitism and Allee effect. J. Biol. Dyn. 4(2010) 86-101.

[24] F.M. Hilker, M. Langlais, H. Malchow, The Allee effect and infectious diseases: extinction, multistability, and the (dis-)appearance of oscillations. Am. Nat. 173(2009) 72-88.

[25] M.E. Wang, M. Kot, Speeds of invasion in a model with strong or weak Allee effects. Math. Biosci. 171(2001) 83-97.

[26] S. Zhou, Y. Liu, G. Wang, The stability of predator-prey systems subject to the Allee effects. Theor. Pop. Biol. 67(2005) 23-31. 
[27] J. Shi, R. Shivaji, Persistence in reaction-diffusion models with weak Allee effect. J. Math. Biol. 52(2006) 807-829.

[28] M. Sen, et al., Bifurcation analysis of a ratio-dependent prey-predator model with the Allee effect. Ecol. Complex. (2012), doi:10.1016/j.ecocom.2012.01.002. 